\title{
Observations of new candidate PMS $\delta$ Scuti stars
}

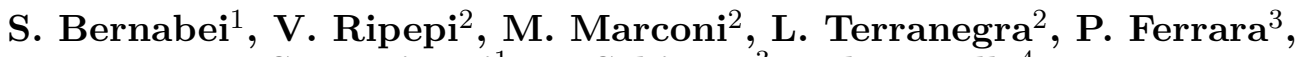 \\ S. Marinoni ${ }^{1}$, E. Schisano ${ }^{3}$ and F. Palla ${ }^{4}$ \\ ${ }^{1}$ INAF - Osservatorio Astronomico di Bologna, \\ email: stefano.bernabei@bo.astro.it \\ ${ }^{2}$ INAF - Osservatorio Astronomico di Capodimonte - Napoli, \\ ${ }^{3}$ Universita' Federico II - Napoli \\ ${ }^{4}$ INAF - Osservatorio Astrofisico di Arcetri - Firenze
}

\begin{abstract}
Among variable stars, $\delta$ Scuti are particularly interesting since they can pulsate in different evolutionary phases and are characterized by both radial and nonradial pulsation modes. Even if the majority of $\delta$ Scuti stars are in their Main Sequence (MS) or post-MS phases, there are growing evidences in the recent years about the presence of $\delta$ Scuti variables among stars which are still in their pre-MS phase (the so-called Herbig Ae-stars). Due to the small number of the known class members, the boundaries of Instability Strip are not well constrained. In this context, we present the preliminary results of a long term project to define these limits by enlarging the sample of PMS $\delta$ Scuti stars.
\end{abstract}

Keywords. Stars: pre-Main-Sequence, stars: oscillations (including pulsations), stars: variables: $\delta$ Scuti, stars: interiors

\section{Introduction}

Marconi \& Palla (1998) derived theoretically for the first time the location of the Instability Strip for intermediate mass pre-Main-Sequence (PMS) stars for the first three radial modes. Their study stimulated the search for $\delta$-scuti type pulsations among the PMS stars. Recently many new members of this class have been discovered (Ripepi et al. 2002, 2003, Marconi et al. 2001, Pinheiro et al. 2003, Ripepi \& Marconi 2004, Zwintz $\&$ Weiss 2004). In this paper we present preliminary results for four new members and three probable candidates of this class.

\section{Observations and data reduction}

The data shown were obtained at the Loiano Observatory 152-cm (Italy) telescope with the BFOSC+CCD or TTCP (3 head photometer) and at the OGS (Teide) telescope. Each CCD dataset has been reduced with standard MIDAS procedures, performing differential photometry between the target star and the respective comparison(s) star(s), while the time is reduced to the Heliocentric JD. On all data sets we performed a Fourier analysis with Period98 (Sperl 1998). Before extracting the pulsation frequencies from the time series, it is important to have a tool to identify the last significant periodicity in the dataset. For this purpose we adopted the empirical criterion suggested by Breger et al. (1993), (see also Kuschnig et al. 1997). These authors state that the signal-to-noise ratio (in amplitude) should be at least 4 to ensure that the extracted frequency is significant. Now we describe our preliminary results for each star. 
Table 1. VVSer frequencies

\begin{tabular}{ccr}
\hline Frequency $\left(\mathrm{cd}^{-1}\right)$ & Amplitude(mmag) & $S / N$ \\
\hline$f 1=5.15$ & 4.6 & 25.3 \\
$f 2=8.61$ & 2.5 & 7.1 \\
$f 3=4.46$ & 2.2 & 7.3 \\
\hline
\end{tabular}

\section{Individual objects}
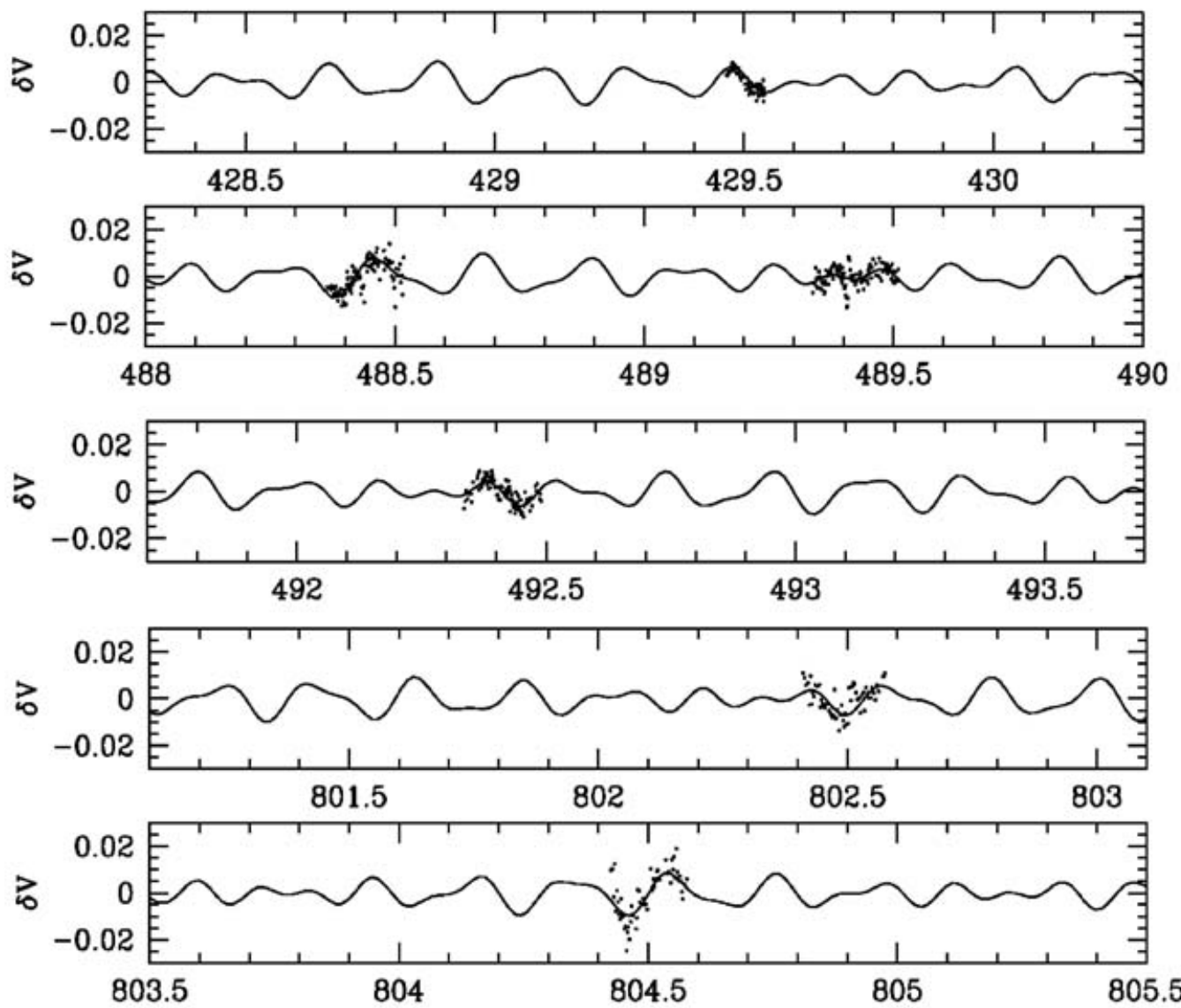

Figure 1. Light curve of VV Ser (points). The meaning of $\mathrm{d} V$ (in magnitudes) is Variable-Comparison. The solid line shows the fit to the data obtained as described in the text.

\section{1. $V V$ Ser}

This star is a Herbig Ae PMS star which is inside the Instability Strip taking into account the data by Chavarria et al. (1988). Photometry of sparse nights from 2001 to 2003 (see Fig. 1) reveals the multiperiodic behaviour of this star. The Fourier analysis show 3 significant frequencies presented in Table 1.

\subsection{V375 Lac}

This star is in a strong reflection nebula that creates some problems in obtaining good photometry, due to the difficulties in separating the contribution of the nebula from that of the star. From the data by Thé et al. (1994), Finkenzeller \& Mundt (1984) and 


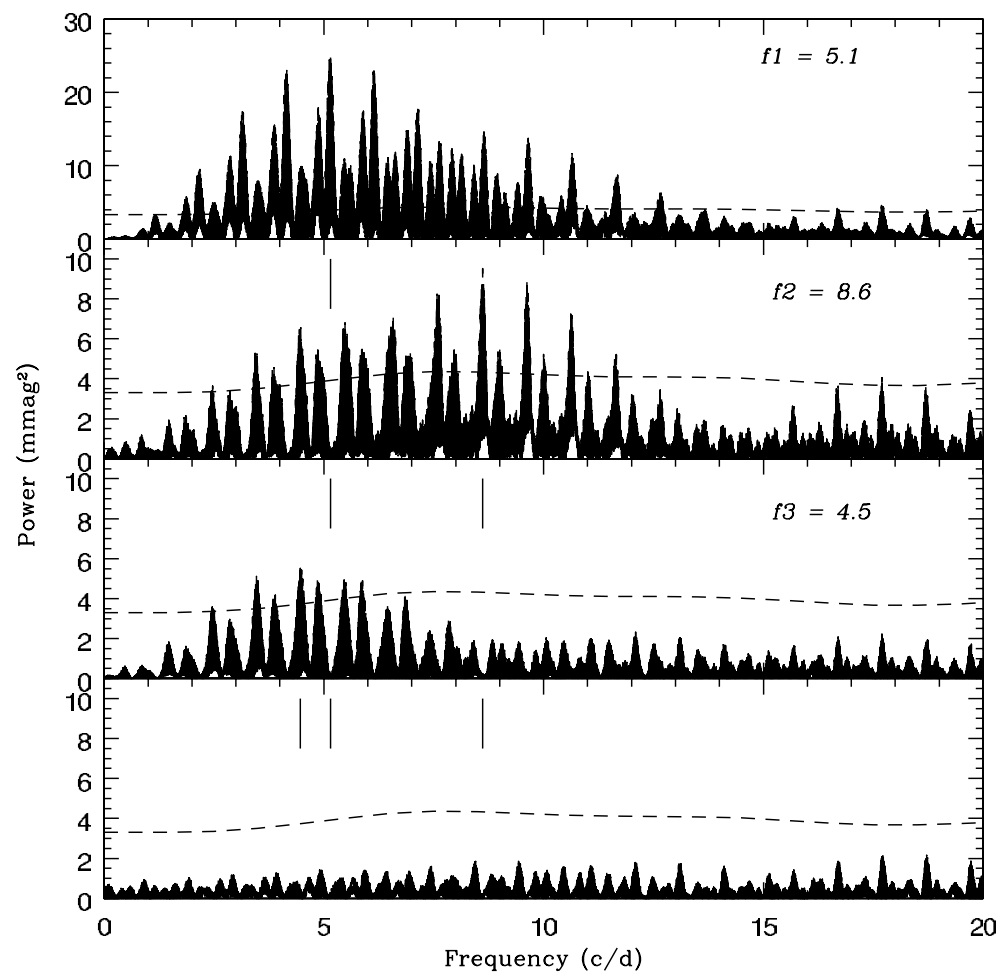

Figure 2. Frequency analysis for the $V$ dataset of VV Ser. Each panel shows the Fourier transform after the subtraction of a pulsating frequency. The dashed line corresponds to $S / N=4$.

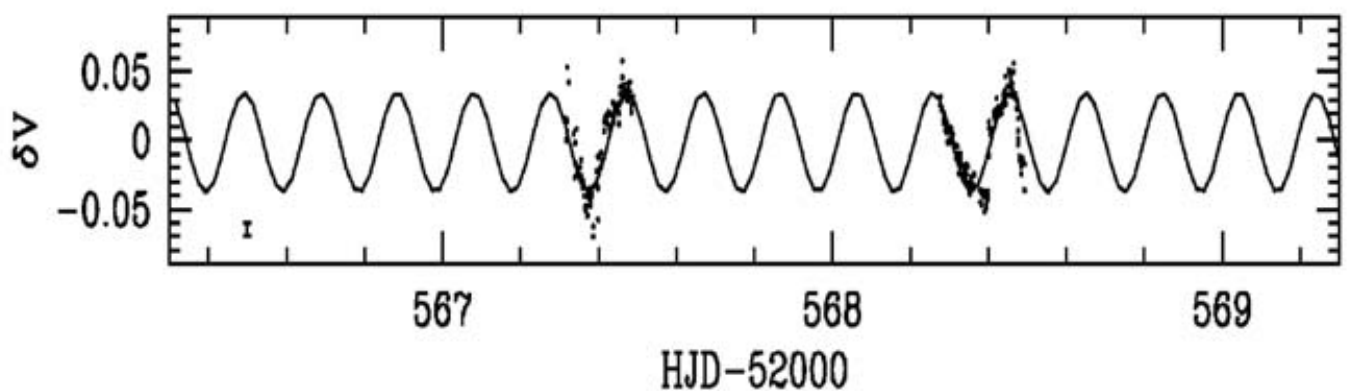

Figure 3. Light curve of V 375 Lac (points). The meaning of $d V$ (in magnitudes) is Variable-Comparison. The solid line shows the fit to the data obtained as described in the text.

For V375 Lac we have two different datasets (CCD and photometer data) that give the same periodicities, but different amplitudes. This is reasonably connected to the presence of the reflection nebula and to the possibility of intrinsic variations of amplitude already discovered in this class. The frequencies found in both data sets are:

$f 1=5.2 \pm 0.5 \mathrm{~d}^{-1}, f 2=9.9 \pm 0.5 \mathrm{~d}^{-1}$. At this moment we can not say if $f 2=2 f 1$ or if it is a real frequency. Thus in our comparison with theory we have considered only $f 1$. 


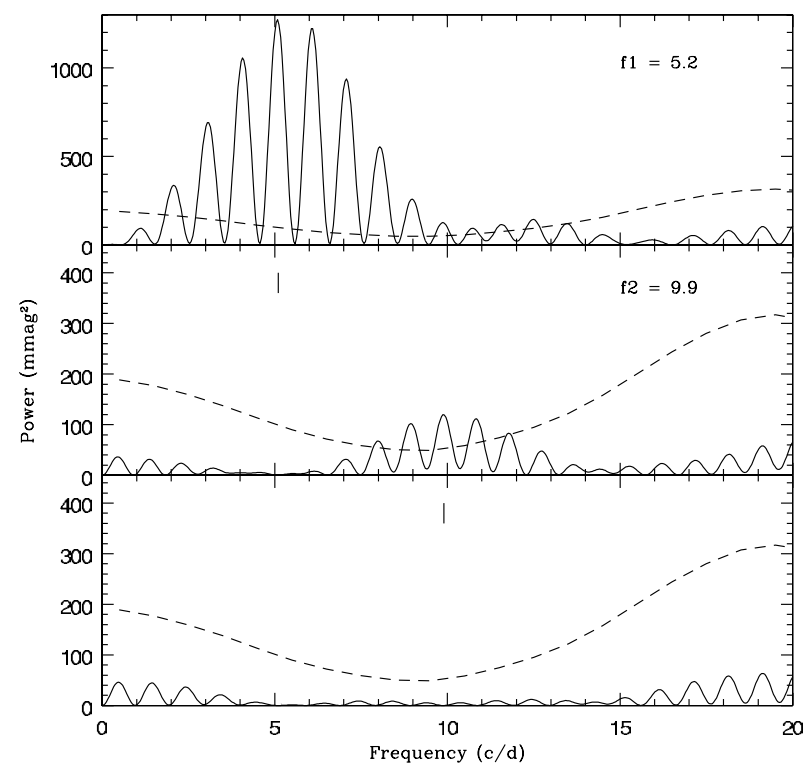

Figure 4. Frequency analysis for the $V$ data set of V375 Lac obtained with the three hannel photometer. Each panel shows the Fourier transform after the subtraction of a pulsating frequency. The dashed line corresponds to $S / N=4$.

\subsection{BN Ori}

From the small dataset that we collected for this star, we can only say that is a pulsating star with at least one periodicity in the range of $10-12 \mathrm{~d}^{-1}$.

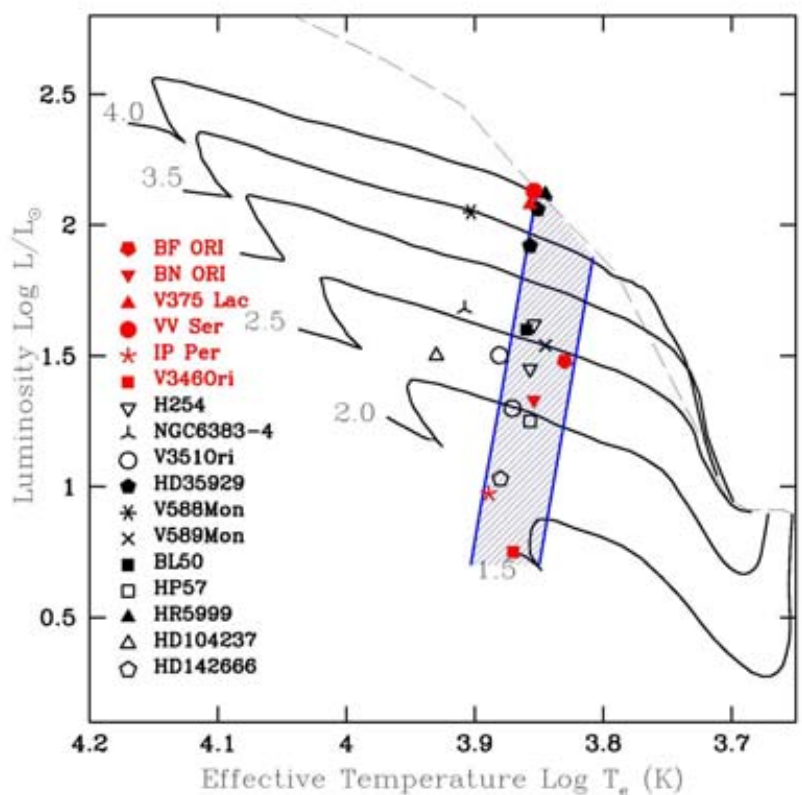

Figure 5. The new Instability Strip. The new PMS $\delta$ Scuti member objects, discussed in this paper, are shown with red symbols. (IP Per is from Ripepi et al. 2005.) 


\subsection{BF Ori}

For this star we can say only that is pulsating at low frequency with $f=4.7 \pm 1.0 \mathrm{~d}^{-1}$.

\section{5. $W W$ Vul}

This star classified as young member of "Orion population" is similar to the intermediate mass star UX Ori. It seems to pulsate at low frequencies $\left(\sim 5 \mathrm{~d}^{-1}\right)$. Only one night of observations has been taken for this star.

\section{6. $P X V u l$}

Although this star is not strictly a Herbig Ae star (the spectral classification is F0V), this star has been investigated because it falls inside the theoretical instability strip. The Fourier analysis show some evidence of two pulsation frequencies: $f 1=5 \pm 3 \mathrm{~d}^{-1}, f 2=$ $13 \pm 3 \mathrm{~d}^{-1}$.

\section{7. $L k H \alpha 168$}

This star result located in the Instability Strip by using the data of Terranegra et al. (1994). One night of photometry seems to show only one frequency: $f \sim 5 \mathrm{~d}^{-1}$.

\section{Comparison with the stellar evolution and the pulsation theory}

Following the same approach adopted in Marconi et al. (2001), we tried to reproduce the $\delta$ Scuti-like frequencies derived in the previous section (only for the VV Ser and V375 Lac for which we have better data) using linear non-adiabatic models of PMS $\delta$ Scuti stars. For each selected mass, the model luminosity and effective temperature have been chosen from PMS evolutionary calculations and the constraint is provided by the observed pulsation period(s). The results are in Table 2 .

Table 2.

\begin{tabular}{lcccc}
\hline Star & $M / M_{\odot}$ & $\log \left(L / L_{\odot}\right)$ & $T$ & Frequencies \\
\hline VVSer & $4.00 \pm 0.25$ & $2.13 \pm 0.02$ & $7150 \pm 25$ & $f 1(\mathrm{TO}), f 2(\mathrm{VII} O), f 3(\mathrm{SO})$ \\
V375Lac & $3.8 \pm 0.2$ & $2.1 \pm 0.2$ & $7194 \pm 25$ & $f(\mathrm{SO})$ \\
\hline
\end{tabular}

\section{Acknowledgements}

We would like to acknowledge the Bologna Observatory for the large observing time allocated for this program at the Loiano Observing Station.

\section{References}

Breger, M., Stich, J., Garrido, R., Martin, B., Jiang, S.Y., Li, Z.P., Hube, D.P., Ostermann, W., Paparo, M., Scheck, M. 1993, A\&A 271, 482

Chavarria-K., C., de Lara, E., Finkenzeller, U., Mendoza, E. E., Ocegueda, J. 1988, A\& $A$ 197, 151

Finkenzeller U. \& Mundt R. 1984, A\&AS 55, 109

Hillenbrand, Lynne A., Strom, Stephen E., Vrba, Frederick J., Keene, Jocelyn 1992, ApJ 397, 61

Kuschnig, R., Weiss, W.W., Gruber, R., Bely, P.Y., Jenkner, H. 1997, A\&A 328, 544

Marconi, M. \& Palla, F. 1998, ApJ 507, L141

Marconi M., Ripepi V., Bernabei S., Palla F., Alcala' J. M., Covino E., Terranegra L. 2001, AEA A 372, L21 
F. J. G. Pinheiro, D. F. M. Folha, M. Marconi, V. Ripepi, F. Palla, M. J. P. F. G. Monteiro,S. Bernabei 2003, A\&SA 399, 271

Ripepi V., Palla F., Marconi M., Bernabei S., Arellano Ferro A., Terranegra L., Alcala' J.M. 2002, A\&A 391, 587

V. Ripepi, M. Marconi, S. Bernabei, F. Palla, F. J. G. Pinheiro, D. F. M. Folha, L. Terranegra, A. Arellano Ferro, X.J. Jiang, J.M. Alcala', T.D. Oswalt 2003, A\&\&A 408, 1047

Ripepi, V. \& Marconi, M. 2004, in: F. Favata, S. Aigrain \& A. Wilson (eds.), Second Eddington Workshop: Stellar structure and habitable planet finding, 9 - 11 April 2003, Palermo, (Noordwijk: ESA Publications Division), p. 397

Ripepi, V. Bernabei. S., Marconi, M., et al. 2005 These Proceedings, GP9

Sperl,M. 1998, Comm. in Astereoseismology (Vienna) 111, 1

Terranegra, L., Chavarria-K., C., Diaz, S., Gonzalez-Patino, D. 1994, A\&AS 104, 315

The, P. S., de Winter, D., Perez, M. R. 1994, A\&AS 104, 315

Zwintz K. \& Weiss W.W. 2004, ISBN 92-9092-848-4, 105 